\title{
Zoom Interface with Dynamic Thumbnails Providing Learners with Companionship through Videostreaming
}

\author{
Takumi Yamaguchi ${ }^{1}$, Haruya Shiba ${ }^{1}$, Masanobu Yoshida ${ }^{1}$, Yusuke Nishiuchi ${ }^{1}$, \\ Hironobu Satoh $^{1}$, and Takahiko Mendori ${ }^{2}$ \\ ${ }^{1}$ Kochi National College of Technology, 200-1 Monobe, Nankoku, Kochi 783-8508, Japan \\ yama@ee.kochi-ct.ac.jp \\ ${ }^{2}$ Kochi University of Technology, 185 Miyanokuchi, Tosayamada, Kami-city, \\ Kochi 782-8502, Japan
}

\begin{abstract}
We have developed the TERAKOYA learning system, which helps students study actively anywhere on a local area network (LAN) linked to multipoint remote users. However, if many students frequently sent their questions to the teacher, it is very difficult to correspond to quickly answer that for the teacher. In addition, the teacher hardly clarifies how much each student understood because he cannot watch students' face and reaction. This paper discusses the graphical user interface (GUI) system that is used a little ingenuity to prioritize students' screens through variably changing the GUI interface on the teacher's PC. The aspect of window that was displayed as thumbnails of the students' PC screen was zoomed dynamically each thumbnail by their understanding level. By sorting out their priorities on the teacher's PC screen, the teacher can timely observe the students' work and support their thinking process.
\end{abstract}

Keywords: GUI, Interactive system, Advanced Educational Environment, Ubiquitous Learning, Distance Education.

\section{$1 \quad$ Introduction}

In today's environment of ubiquitous computers, promoting the use of computers in school is very important. E-learning and learning through web content, however, are passive methods, and it is difficult to cultivate comprehensive active learning, which has recently gained prominence. Because active learning requires learner participation, computers are expected to complement classroom lectures given by teachers to students. Systems for computer-based active learning enable in-class participation by transparently manipulating the input instruments of the students and the teacher. Several researchers have suggested that the challenge in an information-rich world is not only to make information available to people at any time, at any place, and in any form, but to specifically say the right thing at the right time in the right way [1]. In particular, the fundamental pedagogical concern is to provide learners with the right information at the right time and place in the right way instead of enabling them to 
learn at any time and at any place [2]. More importantly, as Jones and Jo [3] pointed out what you, as educators, should aspire to combine the right time and right place learning with a transparent and calm method to allow students to access lessons flexibly, calmly, and seamlessly. Such an approach seems to be a calming technology for the ubiquitous computers, and it adapts itself to students' needs by supporting specific practices.

In the present study, we have developed the new collaborative TERAKOYA learning system [7] for remedial education, which helps students study actively anywhere on a LAN linked to multipoint remote users, as shown in Fig. 1. The TERAKOYA learning system provides both interactive lessons and a small private school environment similar to the 18th-century Japanese basic schools called terakoya. In particular, the system provides an interactive evening lesson that uses tablet PCs on a wireless LAN (WLAN) and custom-built applications that link students in the dormitory and at home with a teacher in the school or at home. In this new system, the students and the teacher cooperate and interact in real time, as in some existing systems, using a personal digital assistant (PDA) [4]. This system can be used to submit and store lecture notes or coursework using a tablet PC.

We define TERAKOYA as a new, evolving virtual private school realized on the network. This makes it slightly different from the 18th-century terakoya. Our TERAKOYA indicates a system for simultaneously achieving the following:

\section{(1) Small group lessons for students, like those at a private school in which the teach-} er becomes the leader.

(2) Interactive lessons that can provide dialog with the teacher and allow students' work to be checked and their thinking processes supported by online collaboration.

(3) Lessons enhanced by mutual assistance that can clarify any misperceptions in the students' thinking processes and provide appropriate support for each student through giving other students' opinions and answers.

In other words, TERAKOYA is an educational support system that can correspond flexibly to the learning demands of many students today by applying a private school model for small group lessons. Therefore, the TERAKOYA system realizes personal learning support for students in a dormitory or at home from a teacher in the school or at home.

In the original terakoya environment, it was not easy to maintain the focus on learning, except for students with a high willingness to learn. On the other hand, our TERAKOYA is a more flexible learning system environment that allows teachers to freely switch between the conventional terakoya environment and the mutually supportive environment using teacher-centered learning or self-paced learning, as needed. Because of this flexibility, we consider that this system can reach a wide range of vulnerable-looking students and accommodate contemporary students' attitudes toward learning. In addition, this system is expected to allow teachers to provide additional learning assistance to students with less additional work for the teacher than supplementary lessons conducted in the classroom or in the dormitory.

We consider a realistic scenario of dynamically providing an interactive lesson for students in an active learning environment in their own living space. As a serious problem in the present system, if many students frequently sent their questions to the teacher, it is very difficult to correspond to quickly answer that for the teacher. In addition, the teacher hardly clarifies how much each student understood because he 
cannot watch students' face and reaction. Accordingly, the GUI of the system uses a little ingenuity to prioritize students' screens through variably changing the GUI interfaces on the teacher's PC. These are blinked, sorted and scaled the students' viewing, etc. In particular, the aspect of window that was displayed as thumbnails of the students' PC is zoomed dynamically each thumbnail by their understanding level. If the PC screens of students can be sorted out their priorities on the teacher's PC screen, the teacher can timely observe the students' work and support their thinking process as an effective teaching aid. The teacher could also clarify any misperceptions in their thinking processes, providing appropriate support for each student.

This paper presents a basic configuration of a system that provides dynamic delivery of full-motion video while following target users in ubiquitous learning environments. The delivery of full-motion video uses adaptive broadcasting. The system can continuously deliver streaming data, such as full-motion video, to the teacher's display as thumbnails of the students' PC screen. Because it maintains the information about the user's attitude in real time, it supports the user wherever he or she is, without requiring a conscious request to obtain their information. This paper describes a prototype implementation of this framework and a practical application.

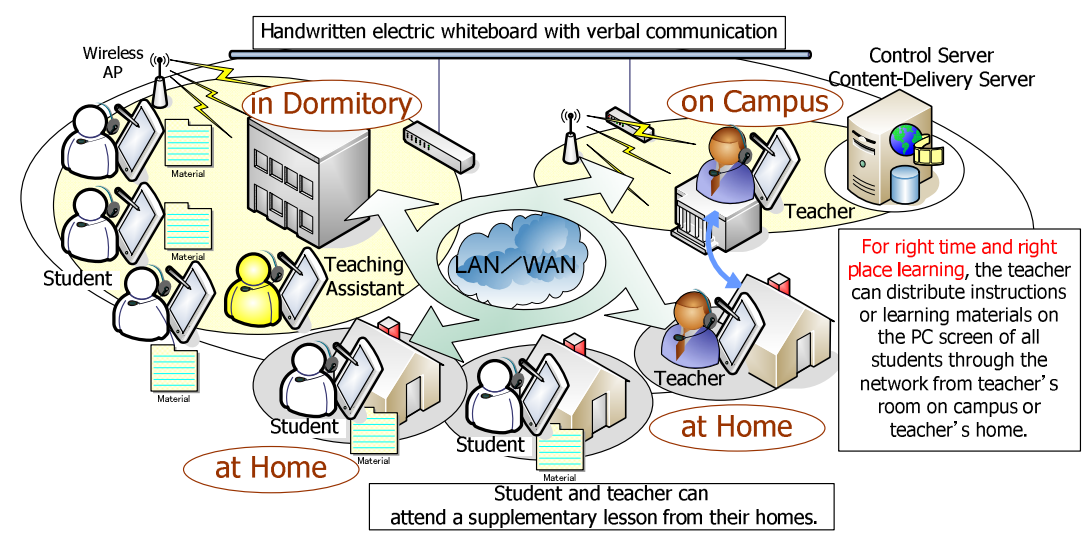

Fig. 1. TERAKOYA learning system

\section{Basic Configuration}

The system consists of tablet PCs, a server machine, and software to enable collaboration among the tablet PCs over a WLAN, which covers the campus, the dormitory, and their homes. The interactive system software consists of server software, authoring software for the teacher, and client software for the students. The authoring software synchronizes with the clients via the server software. The system works in two modes: collaboration mode and free mode. The authoring software is launched on the teacher's PC, which has a 12-inch XGA display. Its main functions are to distribute teaching material, select collaboration mode or free mode, give a specific student's PC the permission to write, view a student's PC screen, share files between a specific student's PC and another PC using the client software, and submit 
coursework using remote control from the teacher's PC. The main functions of the client software are browsing lecture notes, storing learning material, and submitting coursework. Using the authoring software, teachers can view students' PC screens as thumbnails. The thumbnail view can display 50 client PCs. When students submit coursework from their PCs, the filenames are displayed in the order of submission on the teacher's PC. Thus, the teacher can immediately confirm the submission status of a student's coursework.

In the collaboration mode, the display on the teacher's PC is shared by the PC screens of up to 50 students. Each student PC serves as a handwritten electronic board. All students in the class can view the activity of a selected student on their PC screens when that student is completing his/her coursework. Furthermore, students with write access can post discussions on the process of completing the coursework because the teacher can control the ability to write data to each of their PC screens. All students can browse through or view these discussions, resulting in a group discussion.

In the free mode, a student can freely write on the teaching material and coursework sent by the teacher on his/her PC screen. The teacher can watch all students' PC screens, although each student's writing is displayed only on his/her PC screen. If a student cannot complete the coursework, the teacher can provide hints to the student or receive his/her questions by sharing their screens. The displayed content in both modes can be saved on each student's PC or on an external memory device, such as USB memory. Students can freely browse the saved data at any time. When they submit their coursework to the teacher's PC, the teacher can mark it immediately and evaluate it in detail later.

Furthermore, as one possible implementation, the system can include multiple servers, with server software used for data exchange between the teacher's client and that of a student. However, student PCs in the dormitory cannot communicate directly with a teacher's PC connected with another network on the campus because each network is isolated by a firewall. To communicate through client PCs on different networks, at least one control server and a steady network connection via TCP/IP are necessary for the data exchange between a teacher's client and that of a student. By using a server that runs the server software as a control server, our system can provide multipoint remote lessons via connections anywhere on the campus and in the dormitory. Additionally, it can even be accessed from home.

Because interactive lessons are provided, each client must continuously maintain its connection to other computers. Thus, the traffic load between the server and clients grows when the number of connected users increases. Consequently, network hardware must provide adequate system performance for real-time information sharing. This system was optimized to work smoothly between one server PC and 50 client PCs, each with a 12-inch XGA display, for one lesson, and the network speed was maintained at $500 \mathrm{kbps}$ or less for each connection. To limit the amount of data exchange between the client PCs, all teaching material and coursework were sent to the PC screens of all students when each interactive lesson began. After the lesson begins, this system sends only their own written data and the data controlled by the teacher to the students' PC screens.

Although we have already pointed out another problem of this system, if many students frequently sent their questions to the teacher, it is very difficult to correspond to quickly answer that for the teacher. In addition, the teacher hardly clarifies how much each student understood because he cannot watch students' face and reaction. By 
conducting a pre-survey of this system, as freely provided advice in the subjective evaluation, we received useful comments such as "It may take some time before a student's question gets a response from the teacher."

Accordingly, the advanced GUI of the system is configured to use a little ingenuity to prioritize students' screens through variably changing the GUI interfaces on the teacher's PC. These are blinked, sorted and scaled the students' viewing, etc. In particular, the aspect of window that was displayed as thumbnails of the students' PC is zoomed dynamically each thumbnail by their understanding level, as shown in Fig. 2. If the PC screens of students can be sorted out their priorities on the teacher's PC screen, the teacher can timely observe the students' work and support their thinking process as an effective teaching aid. The teacher could also clarify any misperceptions in their thinking processes, providing appropriate support for each student.

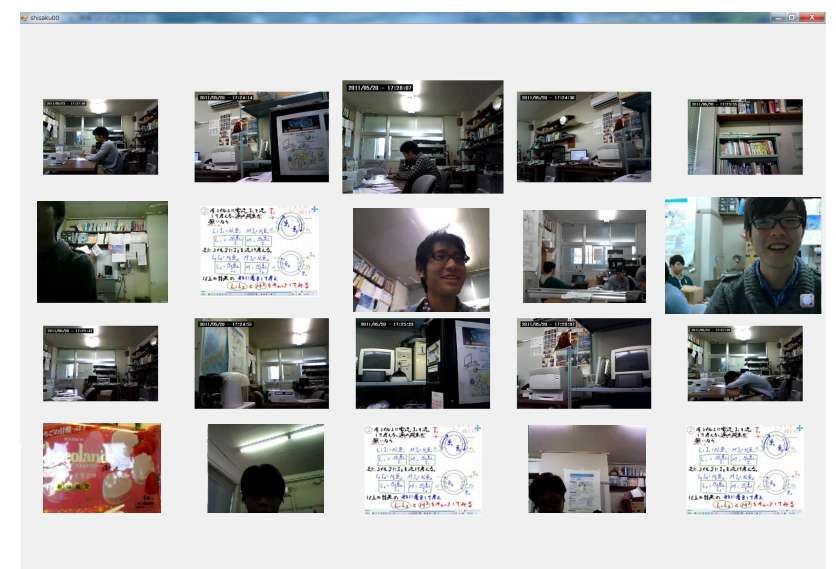

Fig. 2. Overview of GUI interfaces for viewing the students' PC screens as thumbnails on the teacher's PC zoomed dynamically by their understanding level

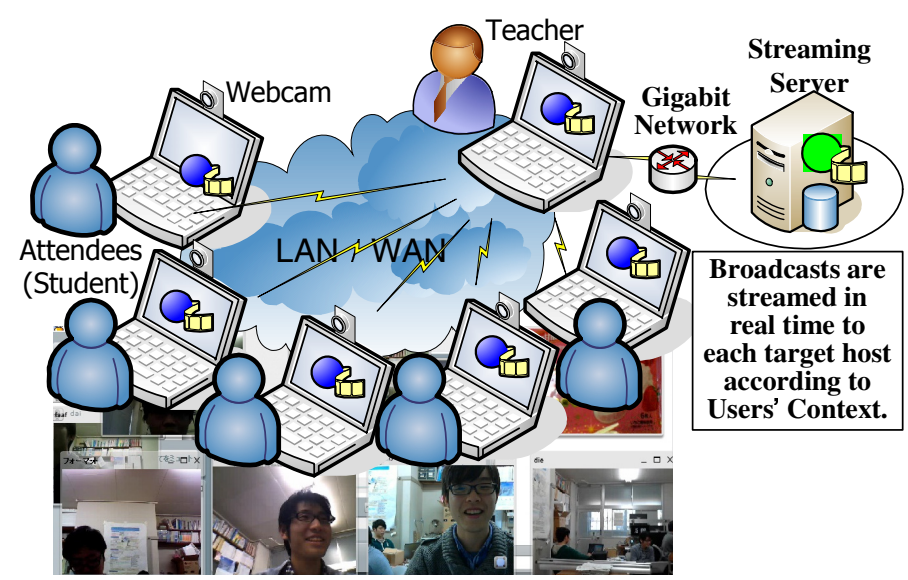

Fig. 3. Experiment of prototype system and its image that we see through five to twenty client hosts 


\section{Practice and Evaluation}

To evaluate the current implementation of our system, we measured its performance in actuating a target host for a broadcast and then measured the response in delivering a streaming video to the whole target host. For this experiment, we used a delivery server and target hosts from five to twenty as shown in Fig. 3. The delivery server ran on a Core i7 $(3.4 \mathrm{GHz})$ processor with Windows 7 Professional and the selfcustomized BigBlueButton 8) with Ubuntu; BigBlueButton is an open source web conferencing system developed primarily for distance education, and supports multiple audio and video sharing, presentations with extended whiteboard capabilities. Each target host ran on a Core i5 $(2.53 \mathrm{GHz})$ Mobile processor with Windows 7 Professional and web browser as a BigBlueButton client. The system interconnects via a Gbit LAN from the server on our campus to an IEEE 802.11g/n WLAN for the target hosts. The streaming video for one target host is played in a $320 \times 240$-pixel (QVGA size) window with a webcam.

We verified the connection speed between the WLAN and the Gbit LAN for the server. When the twenty target hosts for students and the host for the teacher were used in the campus, as shown in Fig. 3, the minimum throughput speed between the server and a host was 12 megabit per second (Mbps). We also measured the time lag before a target host's actuation. The latency from capturing a webcam to passing a streaming server's IP address to a target host was less than $10 \mathrm{~ms}$ and the latency of the web browser's connection between a streaming server and a target host over a TCP connection set a minimum.

On the other hand, we carried out a questionnaire survey to investigate the subjective impression of the prototype system as a subjective evaluation. Test subjects are 10 students of the department of electrical engineering in their twenties. The subjects in their twenties are experienced in the PC operations in their daily life. We explained and demonstrated how to use the prototype system for the subjects before they filled out the questionnaire. After that, questionnaires on the subjective impression were evaluated in a five category rating scale where: Better $=5$, Slightly better $=4$, Fair=3, Slightly worse $=2$, Worse $=1$.

The subjects evaluated this system favorably, which dealt with the system's operability. Because we need to analyze the evaluations of teachers' performances, we would like to discuss the evaluation of the system in more detail later. As the educational effect, the subjects feel that the supplementary lessons using this system had the same effect as a face-to-face class. Moreover, their desire to attend the supplementary lessons in the future increased. The subjects' rating of the useful habit of studying was high because all students answered that the supplementary lessons using this system were more useful for forming the habit of studying, whereas the study time outside of the supplementary lessons was slightly low. Regarding the study time, two students answered "slightly yes," and one student answered "slightly no." Thus, one student had more incentive to study for the course because of the supplementary lessons, and the other student felt that the lessons were sufficient.

These ratings of the supplementary lessons provided by this system suggested that this system could have the same outcome as a face-to-face class if the supplementary lessons are provided as multipoint remote interactive lessons. Also, the evaluated 
value of the prototype system for these users might prove greater with more familiarity and experience with this system.

\section{$4 \quad$ Related Work}

Studies of interactive support systems used in class with a pen-based interface focus on the way in which the system helps the student answer questions and use the teaching material with an electronic board, a PDA, or information and communication technology (ICT) equipment. To compare our TERAKOYA system to related work, we weigh two technical areas: interactive systems [5] and active learning environments, both with pen-based computers [6].

Although each of these related systems is very interesting, ours has the following distinguishing features. First, our system can make it easier for students to ask the teacher questions the same as they could in face-to-face interactions. Second, it is very satisfying for the students that their queries are answered immediately anywhere on a LAN linked to multipoint remote users, which is achieved by directly connecting the teacher and the students. In addition, students may feel a sense of security and of being looked after because the psychological distance between the student and teacher is small. As a result, students can maintain their study concentration longer than in a normal remedial education class. Thus, the TERAKOYA system not only expands the accessibility of popular tablet PC support methods, but also accommodates a wide variety of learning styles by leveraging a transparent, calm learning environment.

\section{Conclusions}

This system helped students study actively anywhere on a LAN linking multipoint remote users and provides an interactive evening lesson using tablet PCs and custombuilt applications both in the dormitory and at home, so students and teachers can stay in their own living spaces. As a prototype, the proposed learning system was implemented in a classroom with a teacher in the teacher's office on the campus. The implementation employed a handwritten electric whiteboard with verbal and nonverbal information. After this test was conducted, the effectiveness of the system in helping students study actively and willingly as an example of "right time, right place learning" was verified.

In addition, ours configured to help the teacher quickly answer students' questions for the teacher. Thus, the teacher can clarify how much each student understood through watching students' face and reaction via a LAN. The GUI system that is used a little ingenuity to prioritize students' screens through variably changing the GUI interface on the teacher's PC. The aspect of window that was displayed as thumbnails of the students' PC screen was zoomed dynamically each thumbnail by their understanding level. By sorting out their priorities on the teacher's PC screen, the teacher can timely observe the students' work and support their thinking process. Using this system, the teacher distributed instructions or learning materials on all the students' PC screens via the network. On receiving this material on their PCs, the students could note their views and answer the questions in the learning material on their PC 
screens using the pens attached to their tablet PCs. They could also submit their answers as an image to the teacher. Because the PC screens of students were viewable on the teacher's PC screen, the teacher could check the students' work and support their thinking processes by online collaboration. The teacher could also clarify any misperceptions by providing appropriate support for each student.

More specific and effective education programs are required. We would like to further evaluate the effect of this system on the understanding and learning motivation of the students when the system is used as an effective teaching aid. We would also like to study the configuration of the new interactive system under active learning conditions. We also would like to further study the configuration of a new interaction system by adding entities. This system is an ambient human interface system, which becomes friendly advisers and gives users naturally awareness, through making real images via a network to follow users. We would like to expect to realize ambient human interface system of a user-oriented ubiquitous computing through implementing this system.

Acknowledgments. We thank Ryuichi Watanabe for his helpful experiments and cooperation. This study was partially supported by a Grant-in-Aid for Scientific Research (C, Area \#1602, Project No. 24501236).

\section{References}

1. Fischer, G.: User Modeling in Human-Computer Interaction. Journal of User Modeling and User-Adapted Interaction (UMUAI) 11(1-2), 65-86 (2001)

2. Ogata, H., El-Bishouty, M.M., Yano, Y.: Knowledge Awareness Map in Mobile LanguageLearning. In: Proceedings of the Sixth IEEE International Conference on Advanced Learning Technologies (ICALT), pp. 1180-1181 (2006)

3. Jones, V., Jo, J.H.: Ubiquitous learning environment: An adaptive teaching system using ubiquitous technology. In: Proceedings of the 21st ASCILITE Conference, pp. 468-474 (2004)

4. Roschelle, J.: Unlocking the learning value of wireless mobile devices. Journal of Computer Assisted Learning 19(3), 260-272 (2003)

5. Miura, M., Kunifuji, S., Sakamoto, Y.: Practical Environment for Realizing Augmented Classroom with Wireless Digital Pens. In: Apolloni, B., Howlett, R.J., Jain, L. (eds.) KES 2007, Part III. LNCS (LNAI), vol. 4694, pp. 777-785. Springer, Heidelberg (2007)

6. Hurford, A., Hamilton, E.: Effects of tablet computers and collaborative classroom software on student engagement and learning. In: Proceedings of Frontiers in Education Conference, FIE 2008, pp. S3J-15-S3J-20 (2008)

7. Nishiuchi, Y., Matsuuchi, N., Shiba, H., Fujiwara, K., Yamaguchi, T., Mendori, T.: Evaluation of TERAKOYA Learning System Linking Multi-point Remote Users as Supplementary Lessons. In: Proceedings of the 18th International Conference on Computers in Education, ICCE 2010, pp. 486-488 (2010)

8. BigBlueButton, http://www.bigbluebutton.org/ 